\title{
A Focus on Biomedical Shape Memory and Superelastic Alloys
}

\author{
Shuichi Miyazaki ${ }^{1}$
}

Published online: 14 March 2016

(c) ASM International 2016

In order for shape-memory alloys to expand their use in biomedical applications, continual research is critical. In this issue of Shape Memory and Superelasticity, several of the articles will be focused on the latest research of welldeveloped NiTi-based and rapidly evolving Ti-based alloys being conducted in both the academic and industrial sectors.

Currently, there is substantially increasing interest in research on biomedical shape memory and superelastic alloys. These materials are NiTi and NiTiX alloys and extending to Ti-based alloys and other classes of materials. Potential studies may include understanding material's functional properties, plasticity, corrosive properties, as well as strength and ductility in addition to materials design and processing techniques to make desired microstructures, shapes, and surfaces. The NiTi-based alloys have developed a big market with many applications both in industrial and medical fields mainly during the last three decades. Rough estimation of the ratio of industrial to medical market sizes is 3-7. Among the medical applications, superelasticity covers more than $80 \%$. The NiTi-based alloys have been successfully used in medical applications. One of the most, if not the most important characteristic is that of biocompatibility. With the severity of health issues that can be posed by the use of materials containing nickel, research is expanding into the area of nickel-free alloys. Hence, Ti-based superelastic alloys have been extensively

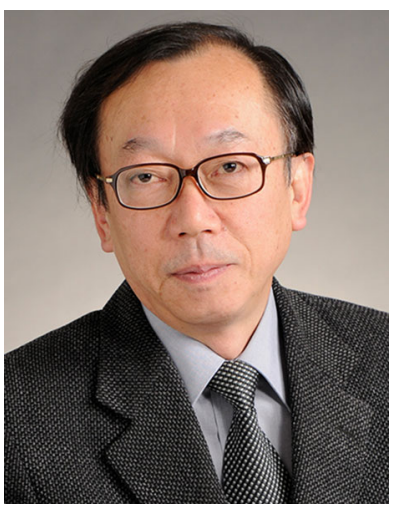

developed during the past 15 years in expectation of expanding the biomedical applications even further. New behavioral and structural properties of these materials are continually being discovered. Processing techniques such as post deformation annealing, analyzing samples through electron microscopy, and testing processes such as tensile tests are continually revealing new properties.

With the articles presented in this issue along with the recent advances in shape-memory alloys research, there is little doubt that these materials will continue to grow in value to the medical world.

Shuichi Miyazaki

miyazaki@ims.tsukuba.ac.jp

1 Division of Materials Science, University of Tsukuba, Tsukuba, Ibaraki 305-8573, Japan 